\title{
Insertion Torque Function Analysis of Novel Dental Implant Geometry
}

\author{
D. Pammer ${ }^{1}$ and E. Bognár ${ }^{1,2}$ \\ ${ }^{1}$ Budapest University of Technology and Economics, Faculty of Mechanical Engineering, \\ Department of Materials Science and Engineering, Bertalan Lajos utca 7., H-1111 Budapest, Hungary \\ ${ }^{2}$ MTA-BME Research Group for Composite Science and Technology, Muegyetem rkp. 3., H-1111 Budapest, Hungary
}

\begin{abstract}
Since the discovery of the osseointegration implant stability has an increasing relevance. Determination of stability is particularly important for dental implants. Two types of stabilities give us information about the success of implantation; primary and secondary stability. There are many stability indicators, but their meanings are not exactly defined theoretically. The aim of our study was to examine and evaluate the insertion and removal torque of novel implant geometry in polyurethane artificial bone blocks with different densities, corresponding to the standard living bone density classification (D1-D4). Protocols given to implantation were followed during the drilling and insertion.

With the analysis of the torque functions we found differences among the polyurethane artificial bone blocks. Torque functions showed us how the function's slope has changed the characteristic max-min values in different polyurethane artificial bone blocks. The drilling parameters highly influenced the characteristic of the torque functions.
\end{abstract}

Keywords - Dental implant, primary stability, insertion torque, novel geometry, artificial bone.

\section{INTRODUCTION}

Measuring the success of the dental implants is an increasing important factor; however there are many key details still unknown in the process of bone regeneration. Since the osseointegration (bone-implant contact) was discovered, one of the most important research topics is to determine and monitor the quality of the implant and bone contact [1].

The measuring of implant-bone integration is called implant stability measurement.

There are two types of stabilities; primary stability and secondary stability. Primary stability is measured immediately after insertion. This indicator estimates the initial success of the implantation. The following main parameters influence the primary stability quality: bone quality - structure and mechanical properties, the implant geometry and the parameters of the drilled cavity. Secondary stability is the result of the bone tissue regeneration and the quality of the contact between dental implant surface and new bones (osseointegration). Secondary stability measurements start after weeks of the implant insertion. The following main parameters influence the secondary stability: dental implant base material, type of the surface modification, bone regeneration ability, the state of health of the individual $[2,3]$.

There are many stability indicators as so many measuring systems to determine it. The nowadays used stability measurement systems have four groups.

The $1^{\text {st }}$ one contains the systems which can measure stability in a non-invasive way. The $2^{\text {nd }}$ one is the group of the invasive measurement systems. The $3^{\text {rd }}$ and the $4^{\text {th }}$ groups show which systems can determine primary and secondary stabilities $[4,5]$. There are also standards for the implant stability measurement. The ASTM F 543 standard contains mostly the biomechanical tests of the bone implants (pullout test, push-in test, driving torque, etc.) [6]. Some type of stability measurements (destructive measurements methods) cannot be used in living bone tissue, due to this there are polyurethane artificial bone blocks.

These blocks are solid foams (ASTM F 1839) [7], with different densities corresponding to the standard living bone densities (D1-D4) [8, 9]. The analysis of a new stability measuring system can be executed in polyurethane artificial bone blocks, cadaver bones and in living or not living animal bones. It is easier to work with artificial bone blocks, because ethical permission is not necessary for them. Artificial bone blocks can be used for the development of a new stability measuring systems as well. The polyurethane artificial bone blocks allow us to compare primary stability of different implant types in vitro and find the ideal geometry with the highest primary stability parameter.

\section{Materials AND METHODS}

\section{A. The aim of the study}

In this study the insertion torque and removal torque measurements are chosen from the different stability measuring methods. The measuring of the insertion torque during the insertion is obvious, because the dental implant must be inserted in the bone. The torque parameters contain a lot of information about the implant, bone properties and about the implantation procedure also.

The aim of this study was to determine insertion and removal torque functions of novel implant geometry in polyurethane artificial bone blocks with different densities. 


\section{B. Materials and instruments}

Polyurethane artificial bone blocks available on the market were used for this research work instead of bone tissue. The use of these bone blocks in vitro is scientifically accepted [10]. Bone blocks with four different densities D1; D2; D3 and D4 were used. Table 1 contains the main properties of these blocks [11].

Table 1 Artificial bone block types

\begin{tabular}{lll}
\hline Artificial bone classes & $\begin{array}{r}\text { Density } \\
\left(\mathrm{kg} / \mathrm{m}^{3}\right)\end{array}$ & $\begin{array}{c}\text { Analogue living } \\
\text { bone densities } \\
(\mathrm{HU})\end{array}$ \\
\hline D1 & $\sim 800$ & $>1250$ \\
D2 & $\sim 640$ & $850-1250$ \\
D3 & $\sim 480$ & $350-850$ \\
D4 & $\sim 320$ & $150-350$ \\
\hline
\end{tabular}

To prepare the bone cavities for the implants the suitable surgical drilling kit and the drilling protocol were used.

Three pieces of Emotion Implant ${ }^{\circledR}$ were used without any surface modification. The novel implant geometry of Emotion Implant ${ }^{\circledR}$ has three main parts (Fig. 1) [12]. The root part has an aggressive large thread just as many implant types on the market. After this, the implant has the novel part, with cylindrical geometry and radial drilled holes. The aim of this part is to increase the surface of the implant with the design itself. The other aim of this part is to collect the bone grist, and facilitate the implant's bone integration. The main sizes of the implant are $12.5 \mathrm{~mm} \times 4.1 \mathrm{~mm}$. The head part of the implant geometry has a small thread as many implant geometries on the market.

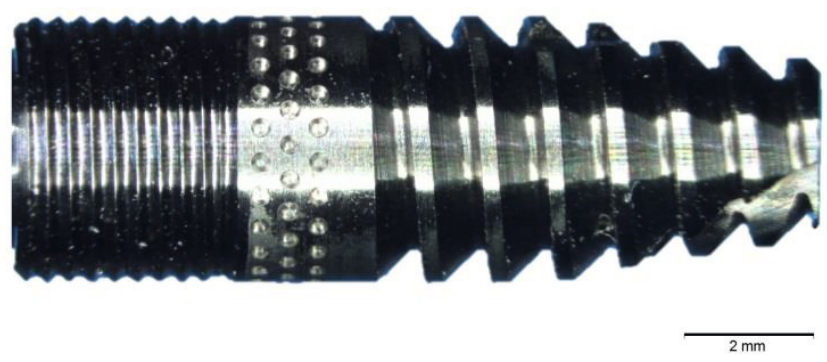

Fig. 1 Emotion Implant ${ }^{\circledR}$

To record the insertion and removal torque values as a function of the rotation angle of the implants an Instron ${ }^{\circledR}$ 5960 tensile test machine (Instron, Norwood, MA, USA) with PTS torque measuring system was used.

For the microscopic analysis an Olympus ${ }^{\circledR}$ SZX16 (Olympus, Tokyo, Japan) stereomicroscope was used.

\section{Methods}

According to the drilling protocol three holes were drilled in each type of artificial bone. Due to this, different hole parameters were observed. The holes in block D1 had the largest diameter, almost the same size as the outside diameter of the implant. Holes in block D4 had much smaller diameter than the outside diameter of the implant, approximately similar to the core diameter of the implant. The holes in blocks D2 and D3 were among the others. By changing the hole sizes, we tried to ensure the best primary stability during the insertion.

The implants were inserted one after the other into the prepared holes (Fig. 2). The insertion speed was 25 RPM, which value is equivalent to the one in clinical use (Nobel Osseocare $^{\circledR}$ ) [13]. During the insertion of the implants the torque was recorded as a function of rotation by the Instron 5960® - PTS system. The same procedure was used for the removal torque measurements as well.

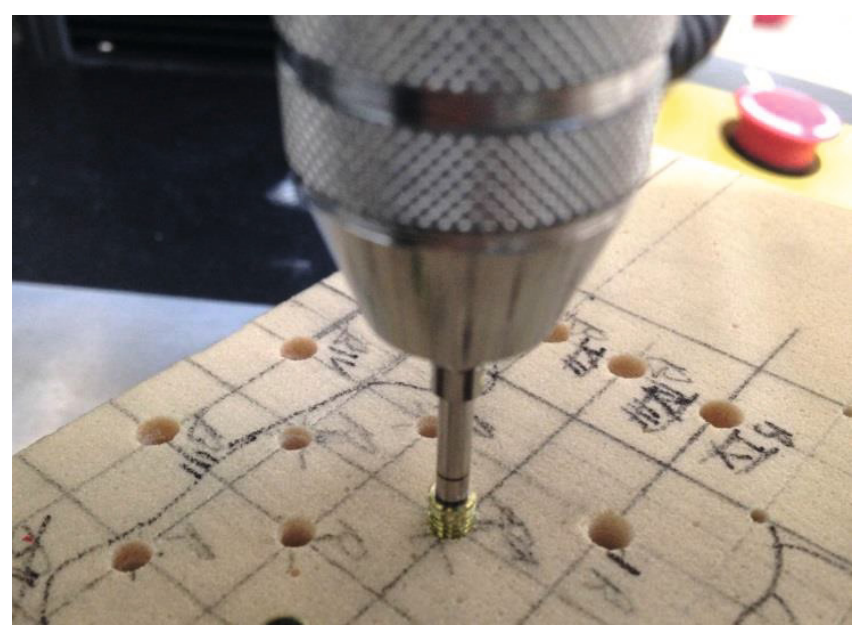

Fig. 2 Insertion of an implant in artificial bone block

Stereomicroscopic images were taken form the screwed out implants, and the cylindrical geometry with radial holes part was analysed. Besides, cross sections were made from implants for the two extreme densities, D1 and D4. The cross section was analysed with the stereomicroscope.

\section{Results}

The microscopic analysis of the unscrewed implant's cylindrical geometry with radial holes shows that the holes are fully filled with polyurethane artificial bone grist (Fig. 3). This phenomenon arose with each implant independent of 
the polyurethane block's densities. This result supported the hypothesis and the expectation due to this novel geometry.
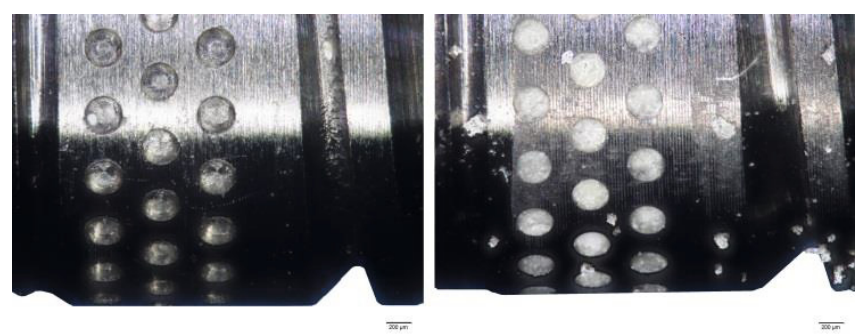

Fig. 3 On the left the empty holes on the implant surface and on the right the holes filled with polyurethane bone grist

The recorded insertion and removal torque functions of the implants were plotted (Fig. 4; Fig. 5; Fig. 6 and Fig. 7) for each block density (D1-D4). With the rotation angle values it was possible to calculate (knowning the implant geometrical parameters) the implant position in the artificial bone blocks. Due to this, the function shows the position associated with the current torque value.

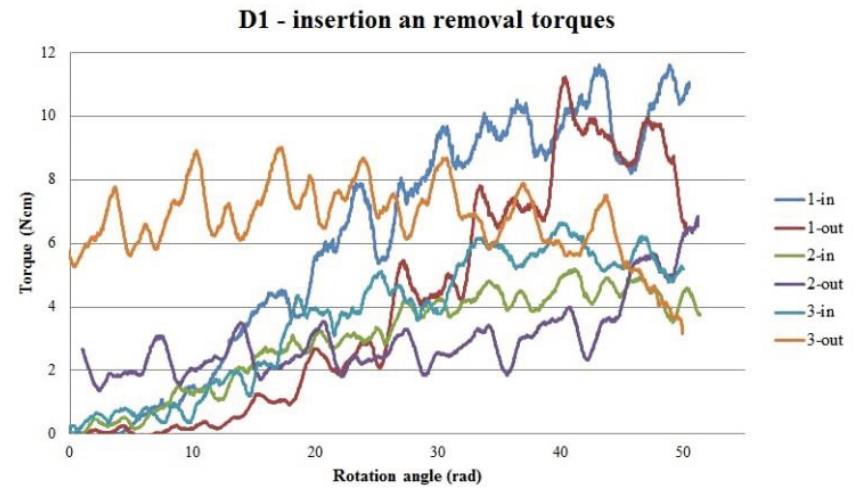

Fig. 4 Implant insertion and removal torque functions in D1 artificial bone

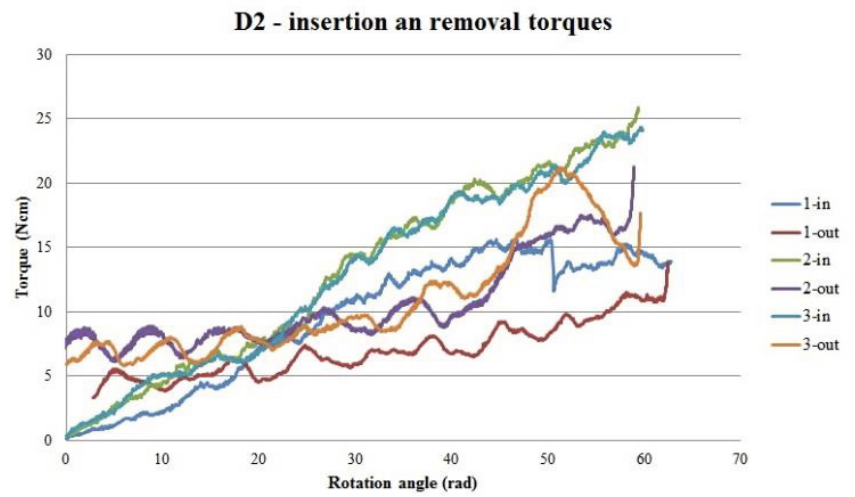

Fig. 5 Implant insertion and removal torque functions in D2 artificial bone
According to the torque functions the results can be divided into two groups. The results of blocks with D1, D2 and the results with D3, D4 densities.

The functions of D1 and D2 show that the insertion torques highly depend on the diameter of the drilled holes. Due to this the minimal size changes in the hole diameters effected totally different torque functions. In the high density polyurethane bone blocks (D3 and D4) the results were influenced by the friction. Due to the large hole diameter the implants were minimally guided by the treads, and minimal deformation was observed in the polyurethane blocks (Fig. 8). The holes with large diameter showed that during the unscrewing the implants did not come out fully from the hole, but further turned in. The removal torque function shows this effect in D1 and D2, where it has a limit, beyond which the function doesn't decrease due to the torque of the friction.

The functions of D3 and D4 show consistently increasing torques as a function of the rotation. The nearly smooth functions show that the polyurethane artificial bone blocks have a homogeneous structure.

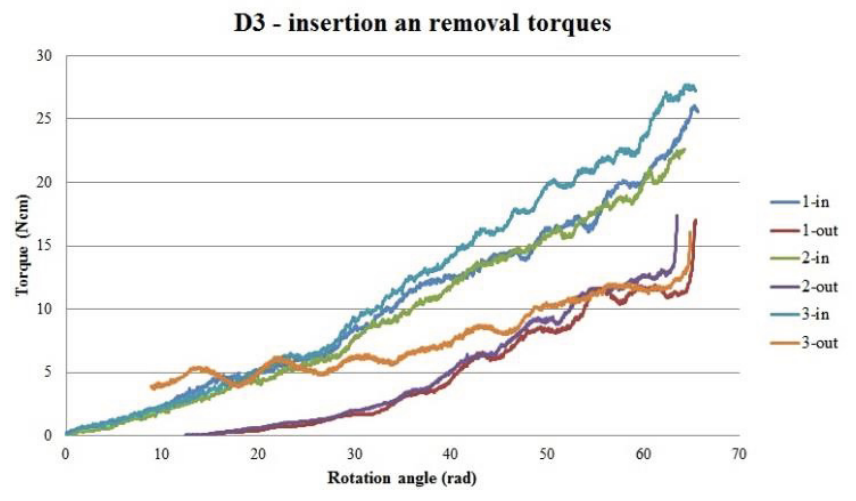

Fig. 6 Implant insertion and removal torque functions in D3 artificial bone

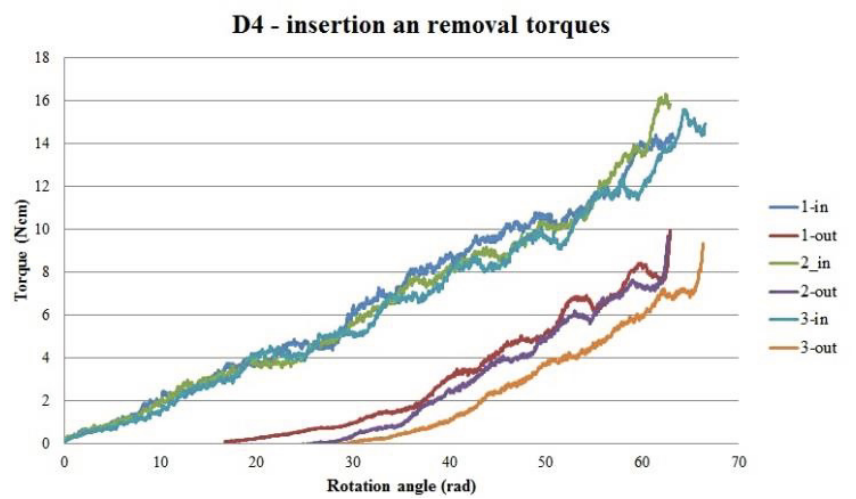

Fig. 7 Implant insertion and removal torque functions in D4 artificial bone 


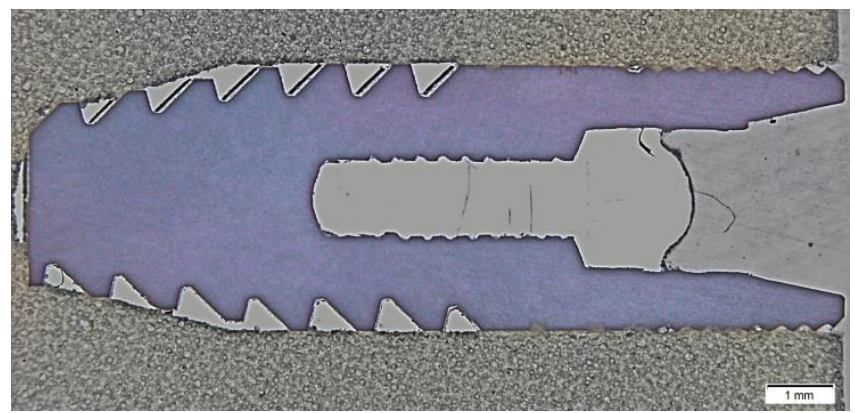

Fig. 8 Implant cross section in D1 artificial bone

Due to the small diameter of the drilled hole the thread guide during the insertion of the implant and the polyurethane artificial bone block had a large deformation near the implant (Fig, 9). Therefore, during the unscrewing the removal torque functions show a continual decrease until the functions reach the $0 \mathrm{Ncm}$ torque value. The microscopic analysis of the cross section allows determining the deformed region near the implant of the polyurethane artificial bone blocks. The two extreme densities D1 and D4 cross section exposures show the different structural changes during the insertion. The deformation of the D2 and D3 blocks are in between the D1 and D4 deformations.

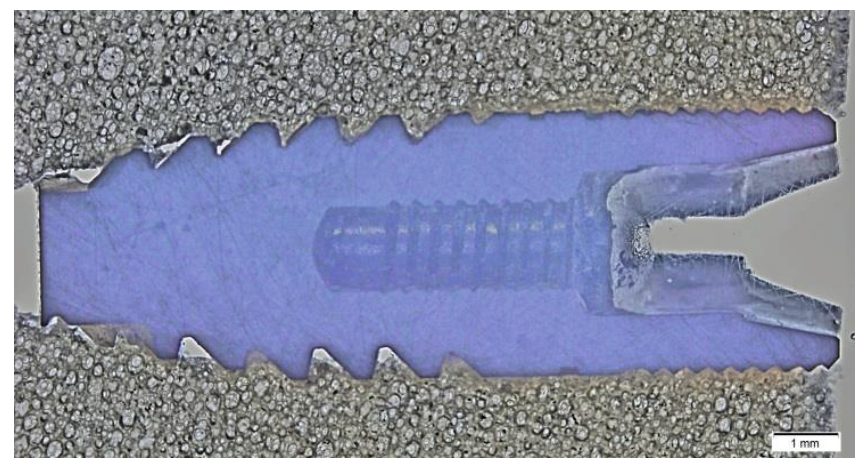

Fig. 9 Implant cross section in D4 artificial bone

\section{CONCLUSION}

The functions of the insertion and removal torques gave us information about the geometrical parameters of the implant and about the artificial bone material parameters. Moreover the insertion torques give information about the inserted implant primary stability. The implant stability and the torque function characteristic are highly influenced by the drilled holes' parameter (diameter) in D1 and D2 polyurethane artificial bone blocks. The stability and the function characteristic are not sensitive to the small differences of the drill diameter in D3 and D4. Due to this the use of this nov- el implant geometry is recommended to D3 and D4 living bone types.

\section{ACKNOWLEDGMENT}

The used implants were provided by Radix Kft.

\section{CONFLict OF InTEREST}

The authors declare that they have no conflict of interest.

\section{REFERENCES}

1. Adell R, Lekholm U, Rockler B, Brånemark PI (1981) A 15year study of osseointegrated implants in the treatment of the edentulous jaw. Int J Oral Surg. 10(6):387-416

2. Elias CN, Rocha FA, Nascimento AL, Coelho PG (2012) Influence of implant shape, surface morphology, surgical technique and bone quality on the primary stability of dental implants. $\mathrm{J}$ Mech Behav Biomed Mater, 16:169-80

3. Chang, P. C., Lang, N. P. \& Giannobile, W. V (2010) Evaluation of functional dynamics during osseointegration and regeneration associated with oral implants. Clinical oral implants research 21: 1-12.

4. Mistry G, Shetty O, Shetty S, Singh RD (2014) Measuring implant stability: A review of different methods. J Dent Implant 4:165-9

5. Kim Y-S, Lim Y-J (2011) Primary stability and self-tapping blades: biomechanical assessment of dental implants in medium.density bone. Clin. Oral Impl. Res. 22, 1179-1184 DOI 10.1111/j.1600-0501.2010.01089.x

6. ASTM F543-00, Standard Specification and Test Methods for Metallic Medical Bone Screws, ASTM International, West Conshohocken, PA, 2002, at http://www.astm.org

7. ASTM F1839-08(2012), Standard Specification for Rigid Polyurethane Foam for Use as a Standard Material for Testing Orthopaedic Devices and Instruments, ASTM International, West Conshohocken, PA, 2012, at http://www.astm.org

8. Misch CE (2007) Contemporary implant dentistry. 3rd ed. Mosby Elsevier. St. Louis

9. Misch CE, Judy KW (1987) Classification of partially edentulous arches for implant dentistry. Int J Oral Implantol. 4(2):713.

10. Bardyn T, Gédet P, Hallermann W, Büchler P (2009) Quantifying the influence of bone density and thickness on resonance frequency analysis: an in vitro study of biomechanical test materials. Int J Oral Maxillofac Implants 24(6):1006-14

11. Sawbones at http://www.sawbones.com

12. Nagy P, Joób F. Á, Schindler Á, Pammer D, Bognár E (2014) Surface modification of dental implants. Biomechanica Hungarica 7(1):42-49

13. OsseoCare Pro at http://www.nobelbiocare.com

Author: David Pammer

Institute: Budapest University of Technology and Economics, Department of Materials Science and Engineering

Street: Bertalan Lajos utca 7

City: H-1111, Budapest

Country: Hungary

Email: pammer@eik.bme.hu 